\title{
Risque et acteurs au travail
}

TIC et dislocations des relations. L'exemple d'un nouveau dispositif numérique (SIH) dans un centre hospitalier privé

\section{Clément Gravereaux et Catherine Loneux}

\section{(2) OpenEdition}

12 Journals

Édition électronique

URL : http://journals.openedition.org/communicationorganisation/4503

DOI : 10.4000/communicationorganisation.4503

ISSN : $1775-3546$

Éditeur

Presses universitaires de Bordeaux

\section{Édition imprimée}

Date de publication : 1 juin 2014

Pagination : 73-86

ISBN : 978-2-86781-904-9

ISSN : 1168-5549

\section{Référence électronique}

Clément Gravereaux et Catherine Loneux, « Risque et acteurs au travail », Communication et organisation [En ligne], 45 | 2014, mis en ligne le 01 juin 2017, consulté le 19 avril 2019. URL : http:// journals.openedition.org/communicationorganisation/4503; DOI : 10.4000/ communicationorganisation.4503 


\section{Risque et acteurs au travail \\ TIC et dislocations des relations. L'exemple d'un nouveau dispositif numérique (SIH) dans un centre hospitalier privé}

Clément Grovereoux' et Cotherine Loneux ${ }^{2}$

\section{Organisation hospitalière et TIC}

\section{Un espace d'émergence de RPS ?}

La notion de risque et la place des TIC dans les processus de travail numérisés, peut selon nous être reliée à une question forte en communication organisationnelle, celle du lien entre risques psycho sociaux (RPS) et changement lié à l'intégration de systèmes d'informations. Elle sera ici interrogée sous l'angle des représentations et des discours propres aux acteurs en contexte de travail, dirigés par le management par la qualité. Cette question du RPS sera travaillée en lien avec les représentations issues de pratiques de travail intégrant des outils numériques, qui conduisent les acteurs à adopter des comportements différents, et qui contribuent à la production de formes culturelles "groupales " (Martucelli) liées aux pratiques professionnelles, formes nouvelles étant potentiellement source de "dislocation » entre les personnels.

Les RPS constituent un sujet de recherche assez innovant tant chez les professionnels que chez les universitaires. Les études et analyses scientifiques sur ce sujet révèlent une traduction physique, physiologique et psychique, une illustration en quelque sorte de l'activité organisationnelle. C'est donc un champ initialement investi par les psychologues et psychosociologues, qui ont permis d'apporter un éclairage remarquable au SIC en ouvrant à des perspectives réflexives sur les conditions organisationnelles d'émergence des RPS. C'est dans cette perspective que nous inscrivons aujourd'hui notre publication. Nous proposons une tentative d'une typologie du risque

1 Clément Gravereaux, PREFics EA4246 : Doctorant en SIC. Ses recherches portent sur la thématique de l'appropriation de système ; clement.gravereaux@gmail.com

2 Catherine Loneux, PREFics EA4246 : Professeure en Science de l'Information et de la Communication, Université Rennes 2. Ses recherches portent sur les communications organisationnelles ; catherine.loneux@univ-rennes2.fr 


\section{$\mathrm{C} \& O \mathrm{n}^{\circ} 45$}

organisationnel dans sa dimension SIC, soit des éléments d'explications permettant de saisir des situations organisationnelles venant ensuite révéler les RPS traités par la psychologie.

\section{Risques et Safe Society}

Les organisations sont censées dans cadre de l'idéologie de la Safe Society. Qu'entend-on par cette notion3 ? Le risque, la sécurité physique (le bienêtre au travail), la sécurité financière (les investissements, la recherche et le développement, les salaires), les risques sociaux (l'anomie, la dégradation des rapports interindividuels au travail). La Safe Society dans le contexte organisationnel se rattache selon nous à la notion de Responsabilité Sociale des Entreprises. Une approche normée des procédures qualité vient anticiper le risque, et créer de fait une représentation anxiogène dans une hypothétique émergence. Elle est une prescription formelle ou informelle qui touche aux usages et aux pratiques de travail, et qui se détache des situations de travail réelles. Le discours organisationnel porté autour du risque fait ainsi partie des interrogations de recherche portant sur l'éthique, et nous interroge sur le plan de la recherche liée aux questions d'identité organisationnelle. Les organisations sont donc traversées à notre sens par des questionnements liés à Safe Society. Nous allons essayer de savoir comment la société du risque et de la sécurité influence les organisations.

Le phénomène sociétal du risque et de la sécurité contamine en quelque sorte les organisations de façon irrationnelle. Cependant, les médias se saisissent de ces questions de risque et de sécurité en les dramatisant. Ceci se traduit dans l'opinion publique par une recherche de témoin de confiance, de rassurance. Les instances normatives comme la Haute Autorité de Santé, obligent les établissements de santé à se soumettre à des normes venant cadrer les processus organisationnels et d'élaboration de stratégie de soin. C'est dans cette forme de traduction du risque par la norme que nous proposons en $\mathrm{SIC}$ une approche particulière du risque, les $\mathrm{RO} 2 \mathrm{i}$ : risques organisationnels impactant l'individu. Nous proposons un raisonnement inversé, partant des RPS pour trouver leur source dans les RO2i.

\section{Essor des TIC et observation des recompositions de l'organisation}

Les formes culturelles et sociales induites par les TIC participent à une fracture des usages, c'est ce que nous avons pu observer dans l'organisation pré citée. Cette fracture est présente, elle n'est pas à prendre au sens extrême du terme, mais dans le sens de transition entre pratiques traditionnelles et pratiques recomposées. Le corps médical avec ses différentes spécialités est un groupe social à la fois très solidaire, soudé, presque familial parfois. Nous

3 Un exemple assez trivial, lié à la sécurité, au principe de précaution : pendant un certain temps, il ne fallait dans les hôpitaux et les cliniques en aucun cas utiliser un appareil téléphonique, risque de dérégler les appareils médicaux... Finalement, ce risque a été identifié comme nul. 
parlons de fracture de transition, car cette propriété intrinsèque de solidarité permet une expression très volontaire de ce que l'on nomme « l'organisation apprenante ». Lintégration des TIC dans le système est liée à la question de l'apprentissage du système d'information hospitalier par les acteurs, ce $\mathrm{SIH}$ devenant progressivement partagé. Un groupe de référent(e)s est formé par la cellule de direction fonctionnelle, puis les référents forment à leur tour d'autres membres de l'équipe de travail, transformant ainsi les modalités de l'apprentissage par des façons de faire individuelles. Cet ensemble constitue pour l'observation une réelle ressource.

Dans certains services, cette forme d'apprentissage organisationnel prend la forme d'un groupe de travail dédié aux échanges, des " trucs et astuces » apparaissent, strictement réservés au partage des pratiques professionnelles sur le $\mathrm{SIH}$, des «fascicules » d'utilisation sont produits. Cette innovation technologique peut être considérée comme une fracture numérique « de transition ", dans la mesure où dans ce contexte précis, l'usage de l'outil ne rencontre que peu de réfractaires parmi les membres du corps paramédical.

Loin de la notion de risque à proprement parler, à l'occasion d'entretiens menés avec plusieurs personnels de l'encadrement et de la direction, nous identifions malgré tout un léger risque de dislocation, de perturbation du social en organisation. Une source potentielle de dérégulation et de ralentissement provient en effet parfois d'une dissonance dans les processus organisationnels, et plus récemment dans les processus informationnels. La notion de risque organisationnel impactant l'individu (RO2i) apparaît ici comme partiellement présente dansl'organisation observée, ce qui nous conduità penserl'organisation en termes de problématique culturelle également, l'organisation apparaissant comme englobant un ensemble d'acteurs rencontrés. Nous parlerons dès lors de risque de dislocation culturelle (liée à des transformations de pratiques professionnelles) intra organisationnelle, mineur, mais présent. Au-delà des RPS et des discours sécuritaires, nous verrons que le RO2i est un facteur de dérégulation de ce qu'il convient de nommer « l'être professionnel au travail ».

\section{L'organisation hospitalière, une proposition de mise en perspective conceptuelle}

Les activités d'un centre hospitalier privé sont codifiées, normées, régies par des instances institutionnelles normatives. Ces instances valident des certifications pour des établissements de santé venant défendre leur performance, pour tout ce qui touche au respect des normes et procédures opérant dans les processus de gestion et de stratégies de soin.

D'un point de vue anthropologique, l'organisation de santé observée ici constitue pour le chercheur un véritable microcosme sociétal. D'une part en effet, dans cette structure hospitalière, travaillent non seulement des professionnels de soin, mais évoluent aussi des patients, des dirigeants, du personnel de services. Une multitude d'activités concourent au fonctionnement du système, et les individus en sont à la fois une ressource et des acteurs. D'autre 
part, nous sommes aussi face à une entité organisationnelle globale, le CHP, et à l'intérieur des cabinets libéraux, ayant au sein même de l'organisation eux aussi, leurs propres modes de fonctionnement processuels et culturels liés aux pratiques à la fois du soin mais aussi de sa gestion.

De façon générale, en dehors des progrès de la médecine, les établissements de santé, se sont équipés de progiciels de gestion intégrée, dénommés Systèmes d'Information Hospitaliers (les SIH). Au-delà des contingences suscitées par les transformations des activités engendrées par l'outil, ce dernier répond directement à un besoin pour les établissements de santé de se conformer aux exigences juridiques, normatives et protocolaires de la traçabilité des actes de soins, de l'accès aux patients de ses informations, du respect des normes dans les stratégies de soin, du suivi des actes, de l'identito-vigilance, etc. L'outil $\mathrm{SIH}$ est aujourd'hui au cœur du processus d'informatisation du dossier de soin.

Du point de vue scientifique, notre proposition est de considérer cet outil comme un dispositif, au sens foucaldien du terme. En effet, ce qui nous intéresse est ce qui se trouve autour de l'outil, c'est-à-dire toutes les recompositions qu'il engendre dans l'organisation (au niveau structural, communicationnel, organisationnel, chez les acteurs en termes de comportement et de logiques d'action ainsi qu'au niveau des pratiques de travail qu'il recompose). On s'aperçoit rapidement que les problématiques soulevées par ce dispositif ne sont pas que liées aux questions de l'utilisation de ce SIH, mais que ce dernier fait remonter également des problématiques organisationnelles sousjacentes. Le SIH apparaît comme une sorte d'actif révélateur de dissonances organisationnelles à tous les niveaux.

\section{L'acteur et l'outil}

Le SIH nous amène à caractériser les recompositions des activités de soin, soignantes et administratives, en « situation de travail ». Les relations interindividuelles et celles avec l'organisation se trouvent ainsi modifiées, le contact entre soignants évolue vers une dimension plus numérisée, dans la façon de s'échanger des informations de santé, de les distribuer dans des flux. Par ailleurs, la réfraction à la traçabilité numérique sous-tend des interrogations liées à la responsabilité, entendue comme " physique ", l'espace de travail interfacé ayant pour conséquence de transposer et de prolonger la présence du patient à l'intérieur de cet espace. Sur ce point, nous parlons de réfraction, non pas vis-à-vis de la démarche qui consiste à tracer numériquement de l'information, mais plutôt en lien avec les représentations de ce changement qui sont offertes par les acteurs en charge de ces outils. Là encore, on peut constater que le paramédical est davantage enclin que le médical à embrasser ce changement de pratiques allant vers de «l'information patient et soin ».

Du point de vue technophile ou technophobe, les SIH et les TIC sont censées améliorer ou détériorer les relations interindividuelles au sein des 
organisations. Dans les deux cas de figure, le risque est de nature sociale, et peut être identifié sous forme de dislocation, ou encore, à l'inverse, sous forme de surinvestissement social, cause de sérieux troubles dans la régulation des activités que les gestionnaires ont du mal à canaliser. Très concrètement, entre médical et paramédical, cette dislocation face à l'usage et la pratique du SIH est visible, des tensions entre ces deux corps de métier émergent. Les infirmières par exemple peuvent retenir une information concernant un patient voire ne pas préparer un patient, pour obliger un praticien à utiliser le SIH. On peut percevoir ce surinvestissement au niveau de l'intégration des équipes dans le déploiement de l'outil, qui finissent par s'investir très sincèrement et proposent, beaucoup voire trop de demandes d'amélioration, de modules complémentaires. Ceci a pour conséquence de créer une frustration par rapport au développement du logiciel qui n'avance pas au rythme des propositions, et typiquement, c'est la confiance même dans le projet global de numérisation de l'information qui est finalement impactée. Nous apportons ici une précision sur la prétendue révolution engendrée par un outil numérique de travail. Déjà cette idée d'outil est un peu confuse. Au sens matériel du terme, l'outil est l'ordinateur, la tablette. D'un autre point de vue si l'outil, le nouvel outil est le nouveau logiciel, quelle que soit sa nature, c'est que l'ordinateur, l'artefact matériel, est intégré, approprié dans le quotidien. Il n'y a plus lieu de parler de révolution, "d'informatisation », ou d'appropriation de l'informatique, le dispositif technique est déjà prolongement de l'action.

C'est là toute la difficulté que l'on a pour décrire ce phénomène d'outillage numérique de l'action en organisation. Est-ce que l'on "informatise " le dossier de soin parce que l'on met dans les services, à disposition des soignants, des PC avec un logiciel pour tracer "l'information patient »? Ou bien serait-il plus judicieux de parler plus simplement d'une modernisation, ce qui permettrait d'ailleurs de mieux comprendre la fameuse résistance au changement qui finalement désigne moins l'appropriation d'un outil que de voir des pratiques traditionnelles professionnelles évoluer ? Bien sûr, l'évolution, la modernisation passe par l'outil, mais dans ce cas-là pourquoi l'idée de résistance à la modernisation par l'intégration d'un SIH dans les processus ne fait absolument pas consensus dans le corps médical, toutes spécialités confondues?

L'outil numérique de travail apporte quelque chose de nouveau aux pratiques de travail, une nouvelle façon de tracer l'information, le passage du papier au numérique. Ou plutôt le passage d'une culture professionnelle liée à la pratique de traçabilité papier à une nouvelle forme, un nouveau support de l'information. L'information numériquement saisie est de nature identique. Cependant, si l'on admet un objet comme support de cristallisation de l'action, le papier conservait une dimension matérielle, palpable, presque « cognitivement » perceptible. L'information numérisée, dématérialisée en quelque sorte, métaphoriquement morte, l'est moins. Pour les soignants, il n'y 


\section{$\mathrm{C} \& O \mathrm{n}^{\circ} 45$}

a rien de nouveau apporté à la tâche primaire : le soin. C'est l'organisation des tâches primaires qui est impactée et transformée par le numérique, pas leur mise en oeuvre.

\section{Une méthodologie de recherche centrée au niveau de l'action située}

\section{Du lieu de travail à la situation de travail}

Le Systèmes d'Information Hospitaliers en organisation hospitalière nous obligent donc à réinterpréter le lieu de travail en situation de travail. $\mathrm{Ne}$ serait-ce qu'en prenant en compte le mail et le smartphone, la terminologie de « lieu de travail » tend à devenir obsolète, et ce tous secteurs professionnels confondus. La « situation de travail " est une bissociation (le terme est emprunté à Arthur Koestler (Koestler, 1964). Elle désigne la cohabitation de deux formes présentielles d'un individu, ici, entre un espace de travail physique et matériel, la Clinique, et un environnement numérique, le système d'information,, traversé par des processualités informationnelles.

Le travail est recomposé par le numérique et marqué par des tendances très fortes de nomadisme (Maffessoli, 1997), transformation majeure de l'essence des activités professionnelles aujourd'hui. L'action ne s'arrête pas au soin, mais à sa traduction tracée, à l'information incluse dans le logiciel, et aux conséquences de cette dernière, soit sur le traitement des informations du patient, soit sur la prolongation de l'action de soin. Toujours en lien avec cette idée de nomadisme, il convient de noter qu'il n'y a plus pour les personnels de contrainte relative au fait devoir être dans son service de soin pour accéder aux « informations patients » que les personnels ont à traiter. Il suffit pour cela de s'identifier sur un ordinateur. L'outil matériel évolue d'ailleurs avec les pratiques, les ordinateurs sont maintenant sur une planche à roulettes accompagnant le soignant n'importe où. Ceci est la même chose concernant les médecins, qui de chez eux, peuvent se connecter au SIH et avoir toutes les informations nécessaires concernant les patients, pour ajuster par exemple leurs prescriptions.

\section{Formes culturelles groupales de pratiques}

Rien ne préexiste à des productions cognitives numériques, est-il habituel de postuler dans les approches communicationnelles des organisations. La forme groupale de pratiques apparaît ainsi comme un construit collectif, qui prend forme sur une base d'échange, de manière à utiliser les systèmes d'information de façon collective. Nous considérons que l'appropriation spatiale d'un environnement au sens organique du terme passe par son appropriation cognitive. Cet ensemble qui participe à l'appropriation du SIH en tant que territoire, nous l'avons vu, passe par la mise en mots, l'individu en étant à l'origine. Tout schème culturel, normatif, social et son usage sont 
témoins d'un niveau supplémentaire d'appropriation, conscientisé ou non d'un environnement de travail.

Il y a donc une corrélation entre appropriation spatiale et production culturo-cognitive, le discours participant de cette dynamique en ce qu'il se mêle aux représentations mentales. On constate au travers de nos observations que des services ou bien des groupes de travail au sein desquels l'appropriation est très forte, se constituent dans ces « lieux » de travail. Les échanges, le partage, la production de connaissances autoémergentes sur l'usage, sont importants. L'action au travail, qui est traversée par le système d'information, prend sens quotidiennement, cette action étant alimentée de significations sur l'intégration de ce nouvel outil. Il reste néanmoins un conflit présent qui est celui de l'adéquation entre la tâche primaire, le soin, et l'utilisation proposée d'un SIH, qui reste apparentée à de l'informatique.

Au-delà de ce lien entre appropriation spatiale de l'outil et production culturo-cognitive, il est important de saisir les évolutions et transformations dans les relations aux pratiques, de cette « culture » de l'information numérique. Elle est en effet saisie, dans son émergence, par les « traces ", les codes, les valeurs, les représentations historiques de l'organisation, etc. En intégrant un nouvel outil ne venant pas compléter la tâche primaire, mais en intégrant directement son organisation, on observe réellement de nouvelles formes culturelles de pratiques globales qui émergent. Certaines pratiques ont cours «naturellement» sur le SIH.

L'utilisation de logiciels de gestion de l'organisation, à quelque niveau que ce soit, va nécessairement entraîner une expression culturelle groupale de pratiques selon nous. De la pratique et des échanges qui opèrent, un groupe est en effet susceptible d'émerger : le groupe des utilisateurs, les formateurs, les référents, les utilisateurs formés par les référents : il y a une hiérarchie d'apprentissage. Chaque niveau d'apprentissage développe ensuite son propre « bricolage » d'usages et de pratiques, une fois confronté au réel, c'est-à-dire en dehors de la situation de formation.

Dans le secteur du soin cette dimension est d'autant plus importante qu'elle laisse la place à certains membres traditionnellement " en bas » de l'échelle du collectif de travail, aides-soignants, infirmiers, de faire entendre leur voix autour de l'utilisation du numérique en situation professionnelle. On observe également des formes culturelles groupales de non pratiques, de réfraction entre autres chez les médecins. On s'aperçoit que ces éléments de réfraction ont peu ou prou à voir avec les capacités du système d'information, mais relèvent davantage de contingences politico-organisationnelles. Cependant, l'appropriation de l'espace numérique est fonction également de son déploiement « culturel », et parallèlement, un déploiement culturel négatif existe aussi. Nous entendons par là, toute production véhiculée sous quelque forme que ce soit, participant à la cristallisation tacite du vécu, de l'expérience négative de l'usage de SIH. 


\section{$\mathrm{C} \& O \mathrm{n}^{\circ} 45$}

Peut-on parler d'une fracture numérique socioculturelle?

R. Kling parle de "Social Access ", qui désigne dans le rapport aux TIC et aux environnements numériques de travail de manière générale (ENT), l'inégalité dans la connaissance et les compétences, comme étant sociale. Ce n'est pas parce que l'accès aux TIC et aux ENT est simple et démocratisé, nous explique l'auteur, que le changement dans les pratiques informationnelles professionnelles va de soi. S'il n'y a pas de prédisposition, d'incitation sociale, l'évolution et l'adaptation au changement des situations de travail, de toute nature seront difficiles pour les usagers. La fracture numérique apparaît comme un écart de pratiques générant des inégalités et entraînant des conflits face au système de management qualité qui impose des normes de pratiques, notamment autour de la traçabilité, qui juridiquement en médecine est une question primordiale et d'ailleurs vécue très positivement.

La modalité normative nous apparaît dans nos observations comme étant dominante à l'hôpital. Nous reprenons sciemment l'idée de "symbolique diabolique » de Fred Poché (Poché, 2009) selon laquelle un dispositif peut produire de la reliance sociale et en même temps de l'exclusion : il y aurait des nantis de l'information (au sens technique du terme). De même, Béatrice Galinon-Mélennec relève : «La diversité sociocognitive des salariés se traduit par une différenciation de leurs compétences dans l'utilisation de l'outil et donc, dans le contenu que l'outil véhiculera » (Galinon-Mélénec, 2010). En effet, il n'y a pas de lien direct entre la possibilité d'accéder à des technologies nouvelles, et ensuite le choix d'en faire l'usage, et encore moins l'usage efficace dans l'organisation. Pour que cela soit possible, encore faut-il posséder les connaissances et compétences cognitives nécessaires et être intéressé par l'outil (De Haan, 2004) - ou du moins s'en reconnaître les capacités (Bandura, 1997).

Nous rejoignons ici Van Djik (2005) : il faut « pouvoir compter sur un support social pour faire face aux difficultés et développer des usages qui permettent de négocier une position sociale valorisante au sein de la société ». Il désigne ainsi les inégalités numériques selon les ressources nécessaires à leur appropriation, dans un premier temps : cognitive, matérielle et sociale et dans un second temps : instrumentale, informationnelle et stratégique.

\section{Proposition de construction d'un objet de recherche portant sur le lien entre l'action recomposée et le risque organisationnel}

\section{Quel lien entre risque et changement?}

On peut poser la question : dans quelle mesure le discours sur les risques et le changement (rapport au principe de responsabilité) ont-ils une influence sur les rapports individuels et collectifs entre acteurs en organisation? Comment mesurer leur influence ? La mesure n'est pas quantifiable, on peut mesurer la portée de cette influence selon l'ampleur des représentations des individus 
avant et après introduction d'un discours du risque et du changement. Estce que les représentations de risque et de changement appréhendées par la branche décisionnelle de l'organisation trouvent matière à appropriation au niveau des acteurs?

\section{Les risques organisationnels impactant l'individu (RO2i), une proposition théorique}

À partir du tryptique : risque/sécurité/qualité on passe aux risques organisationnels.

Quand on parle de risque psychosocial dans la santé, il faut bien percevoir ceux qui sont du «bon côté » du conflit. D'après Crozier et Frieberg (1981), les situations pathologiques, et c'est bien ce que nous cherchons à illustrer par les RO2i, concernent ceux qui sont :

- en position d'infériorité dans les relations de pouvoir ;

- affectés le plus par la crise du sens ;

- les plus touchés par les recompositions organisationnelles.

Ce sont les acteurs concernés par ce tryptique de "symptômes ", qui vivent ces risques organisationnels les impactant (RO2i), et générant de la souffrance. Le risque dans notre appréhension particulière désigne très clairement le risque de situations de travail perdant de leur stabilité. Ce que l'on peut appeler à tort résistance au changement vient de l'élément suivant : dans ce que l'on appelle les logiques d'actions, dans le cadre de l'appropriation des outils et des environnements numériques de travail, le degré d'appropriation et de rejet sera fonction de l'implication de l'acteur au niveau maximum de ce qui ne le mettre pas en situation de faiblesse dans des relations de pouvoir, de négociation. Si un acteur a le sentiment de voir diminuer ce pouvoir, il n'embrassera pas le changement. Autrement dit, les dissonances organisationnelles sont avant tout fonction des incidences des recompositions vécues par les acteurs, l'acteur seul ne provoquant pas de " chaos». La source d'une analyse des organisations aux prises avec les TIC semble une fois encore se situer dans l'analyse de ses constantes recompositions et des effets induits processuellement sur les pratiques. Le risque organisationnel impactant l'individu peut à cet égard être considéré comme une grille de lecture, de prise de hauteur sur des phénomènes de recomposition, visant à les appréhender sous un regard critique.

\section{Des risques organisationnels identifiés dans l'organisation observée?}

En guise de conclusion, nous reviendrons sur nos résultats de recherche à ce stade, issus de nos travaux à la clinique Saint Grégoire. Nous avons pu observer autour des pratiques et usages des TIC des formes d'universalisation processuelle, autrement dit, des processus organisationnels globaux, appliqués à une entité organisationnelle dans son entier sans distinctions de particularités individuelles, de profession, de situation de travail, de condition 
d'usage des outils. Du point de vue des risques éventuels, l'atomisation du collectif par rupture radicale de culture de pratiques de travail peut être considérée comme parfois émergente. L'atomisation interne du collectif par la rupture d'usage et de pratique professionnelle culturelle entraîne ainsi une période de transition qui amène la restructuration des bases de ce collectif. Cette dislocation invisible du collectif peut faire émerger des tensions entre utilisateurs, notamment dans la recherche et l'accès à l'information, ce qui déclenche parfois une souffrance du corps social professionnel.

Toujours du point de vue des risques, nous pouvons identifier également une mutualisation forcée qui viendra bloquer l'appropriation de l'outil. En effet, à l'intérieur d'une structure globale, les cabinets libéraux présents ont leur fonctionnement propre, leur logiciel d'information patient, qui définit une partie de leur identité professionnelle. Ce logiciel introduit à tous les niveaux de l'organisation un outil unique, qui entend mutualiser des individualités qui ne cherchaient pas nécessairement à être réunies. Utiliser un outil unique revient en quelque sorte à mettre des individus de différentes professions, avec des échelles hiérarchiques implicites, sur un pied d'égalité face un outil technologique, une technologie informationnelle unique, et cela génère des freins aux usages.

Un autre risque réside aussi dans la recomposition des compétences des acteurs. Le SIH même intégré dans le quotidien rend difficilement identifiable la dimension informationnelle des compétences des soignants. Le risque est de ne pas identifier à quelle personne s'adresser pour résoudre des problèmes d'ordre techniques : une connexion Wifi, un problème d'impression, un problème d'affichage de données à l'écran, etc. Ce n'est plus la course à l'information, mais à qui aura la compétence pour répondre à ce type de problèmes et, très concrètement, cela fait émerger des tensions, car les soignants ne souhaitent pas être mêlés au statut de technicien informatique. Le fait de fixer l'appropriation des nouveaux outils dans des protocoles soulève un problème de prise en compte de l'évolution des recompositions réelles organisationnelles affublées de protocoles. Les protocoles normatifs d'usages du SIH entrent souvent en conflit avec des situations réelles de travail qui passent au travers de cette standardisation normative, de façon presque instinctuelle.

Un dernier risque est celui de la standardisation possible dans les stratégies de soin, la prescription des protocoles, favorisant l'intercompréhension dans l'échange d'information: il y a risque de produire des standards d'interprétation qui peuvent parfois impacter l'autonomie, le libre arbitre des acteurs, venant les fragiliser.

On peut ainsi postuler que la cohabitation non seulement de la culture informationnelle papier et numérique, mais aussi de la culture sociotechnique de différents logiciels de soin pour différentes pratiques médicales mais existant au sein d'une identité organisationnelle globale, crée des conflits à tous 
niveaux, et empêche d'établir une culture sociotechnique stable, les différents logiciels ne fonctionnant pas de la même façon. Il nous semble important de poursuivre ces travaux sur les risques psychosociaux dans une perspective inscrite en Sciences Humaines et Sociales (Sciences de l'information et de la communication, psychosociologie notamment), et de continuer à approfondir les analyses portant sur les transformations des vécus organisationnels souvent générateurs de crise du sens, cette crise étant accentuée par des représentations et des discours découlant du numérique et de la Safe Society.

\section{BIBLIOGRAPHIE}

BANDURA A, "Self efficacity : towards an unifying theory of behavioural change ", p. 191-215, Psychological Review, (84) 2, 1997.

BARBIER Jean-Marie, "Cultures d'action et modes partagés d'organisation des constructions de sens ", Revue d'anthropologie des connaissances, 2010/1, vol 4, n 1, p. 163-194.

BOURDIN S., BOUILLON J-L., LONEUX C., dirs, " Approches communicationnelles des organisations, Perspectives internationales ", Sciences de la Société, $\mathrm{n}^{\circ}$ 74, Toulouse, Université de Toulouse 3, LERASS, 2008.

BOURNOIS Franck et BOURION Christian, « Devenue une arme idéologique de combat, la RSE introduit de nouveaux risques psychosociaux ", Revue internationale de psychosociologie et de gestion des comportements organisationnels, 2010/38, vol. XVI.

CHOI Hangsub, "Le nomadisme maffesolien et la culture numérique ", Sociétés, 2010/4, n 110 , p. 139-148.

COUTANT Alexandre et STENGER Thomas, «Production et gestion d'attributs identitaires », Les Cabiers du numérique, 2011/1, vol. 7, p. 61-74.

CROZIER M., FRIEDBERG E., L'acteur et le système, Les contraintes de l'action collective, Paris, Éditions du Seuil, Première parution en 1977, dans la collection «Sociologie politique», 1981.

DE HAAN J., " A multifaced dynamic model of the digital divide », p. 66-88, in IT and Society, vol 1, $\mathrm{n}^{\circ}$ 7, Stanford University Press, 2004.

GALINON-MÉLENEC B., "Réseaux sociaux d'entreprise et DRH, Nouveaux outils pour de nouveaux enjeux ?, Communication et Organisation, n $\mathrm{n}^{\circ}$ 37, 2010, p. 41-51.

GEORGES Fanny, "Représentation de soi et identité numérique " Une approche sémiotique et quantitative de l'emprise culturelle du web 2.0, Réseaux, 2009/2 n 154, p. 165-193.

KLEIN Annabelle, Les pages personnelles comme nouvelles figures de l'identité contemporaine, analyse narrato-pragmatique des récits de soi sur internet, publication de l'université catholique de Louvain (thèse), CIACO, 2002.

KLING R., "Technological and Social Access on Computing, Information and Communication Technologies ", White Paper for Presidential Advisory Committee on High Performance Computing and Communications, Information Technology, and the Next Generation Internet, July : http://www.slis.indiana.edu/faculty/kling/pubs/NGI, 1998.

KOESTLER Arthur, 1964, Le cri d'Archimède, Paris, les belles Lettres. 


\section{C\&O n ${ }^{\circ} 45$}

MACHADO DA SILVA Juremir, "Nouvelles technologies, nouvelles socialités, anciens sentiments ", Sociétés, 2006/1 n 91, p. 69-74.

MARC J. et al., "Dynamique cognitive et risques psychosociaux : isolement et sentiment d'isolement au travail », Le travail humain, 2011.

MAFFESOLI Michel, Du nomadisme - Vagabondages initiatiques, Paris, Le Livre de Poche, 1997.

MARTUCELLI D., 2002, Grammaires de l'individu, Paris, GallimardPauly Véronique et Viers Jacques, «L'apport de la sociologie à la prise en compte des risques psychosociaux dans l'entreprise ", Sociologies pratiques, 2008/1.

PERAYA Daniel, « De la diversification des usages sociaux des TIC considérée comme effet et cause d'inégalités sociales ", Distances et savoirs, 2010/4, vol. 8, p. 643-654.

PETIT J. et al., " L' intervention ergonomique sur les risques psychosociaux dans les organisations : enjeux théoriques et méthodologiques ", Le travail humain, 2011/4.

POCHÉ F., "Les défis d'une paix sociale durable face au processus de désymbolisation ? ", p. 101-105, Revue d'éthique et de théologie morale, 2009/3, n 255, Paris, Cerf ed.

Résumé : Le Centre Hospitalier Privé (CHP) de Saint-Grégoire nous offre pendant trois ans la possibilité de suivre le déploiement d'un nouveau système d'information. C'est donc au cour des questions de recompositions organisationnelles traversées par les TIC que nous situons cette proposition. La notion de risque et la place des TIC dans les processus de travail numérisés, peut selon nous être reliée à une question forte en communication organisationnelle, celle du lien entre risques et changement. Cette question du risque sera travaillée en lien avec les représentations issues de pratiques de travail, qui conduisent les acteurs à adopter des comportements, et qui contribuent à la production de nouvelles formes culturelles "groupales » (Martucelli) liées aux pratiques professionnelles et étant potentiellement source de «dislocation ». Dans le cadre de cette réflexion, nous envisagerons donc quel peut être l'apport d'une analyse communicationnelle à la compréhension des représentations et des comportements (Bouillon, Bourdin, Loneux, 2007). Au-delà des Risques Psycho Sociaux (RPS), des discours sécuritaires, etc., nous verrons une autre forme de risque, le risque de dérégulation de l'être professionnel au travail à travers les technologies du soi, idée que nous allons explorer dans cet article , le $\mathrm{RO} 2 \mathrm{i}$ : risque organisationnel impactant l'individu.

Mots-clés : communication, organisation, TIC, système d'information, santé, management, risque, information médicale.

Abstract : The Private Hospital Center of Saint-Grégoire has allowed us to follow, during three years, the implementation of a new information system. This paper gives an insight of organizational transformation issues caused by information and communication technologies. The notion of risk and the role of digital technologies in digitalized workflows, can, according to us, be connected to a key issue in the Communication of Organizations: that of the link between risk and change. This question of risk will be studied in relation with work representations, 
who engage players to adopt new behaviours and take part in the emergence of of new groupal and cultural entities that are linked to work habits and should be considered as a potential source of "dislocation". In this perspective, we will study the contribution of a communicational analysis to the understanding of representations and behaviours. Beyond psychosocial disorder issues and speeches around safety, we will cover another kind of risk: that of work deregulation for human professional in the digital era, issue that will be studied in this article, the OR2i: Organizational Risk Impacting the Individual.

Keywords : communication, organization, information and communication technology, ICT, digital technology, information system, health, management, risk, health information. 
